\title{
The Role of Risk-sharing Mechanisms in Finance Health Care and Towards Universal Health Coverage in Low-and Middle-income Countries of World Health Organization Regions
}

\author{
Ali Ahangar' ${ }^{1}$ Ali Mohammad Ahmadi' ${ }^{2}$, Amir Hossein Mozayani' ${ }^{2}$, Sajjad Faraji Dizaji ${ }^{1}$ \\ ${ }^{1}$ School of Economics and Management, Tarbiat Modarres University, Tehran, Iran; ${ }^{2}$ Economic Research Center, Tarbiat Modarres University, Tehran, \\ Iran
}

The costs of health care are increasing worldwide. Patients are compelled to pay more for treatment, causing many people to fall below the poverty line [1], and one of the most urgent and vexing challenges faced by many low-income and middle-income countries is providing health care for the more than 2 billion poor people who live in such nations. Direct outof-pocket (OOP) payments by patients account for as much as $80 \%$ of total health care expenditures in low-income countries. Studies in several countries have found that significant medical expenditures are a major cause of poverty. In addition, according to a World Bank report [2], only $11 \%$ percent of global health spending corresponded to $90 \%$ of the world's population, with the following distribution among non-developed countries: Asia, 3.5\%; the US, 3.2\%; Europe, 2.4\%; the Middle East and North Africa, 1.5\%; and Africa, 0.4\%; meanwhile, developed countries accounted for $88.9 \%$ of spending [2]. In $2007,87.5 \%$ of the global disease burden was distribut-

Received: December 2, 2017 Accepted: December 19, 2017

Corresponding author: Ali Mohammad Ahmadi, PhD

School of Economics and Management, Tarbiat Modarres University,

Nasr, Jalal AleAhmad, Tehran, Iran

E-mail: aahmadi@modares.ac.ir

This is an Open Access article distributed under the terms of the Creative Commons Attribution Non-Commercial License (http://creativecommons.org/licenses/by$\mathrm{nc} / 4.0 /$ ) which permits unrestricted non-commercial use, distribution, and reproduction in any medium, provided the original work is properly cited. ed in low-income and middle-income countries, but only $12.5 \%$ of global health spending took place in this group of countries. Conversely, in developed and highly developed countries, with a very low proportion of the global disease burden $(12.5 \%)$, the share of total health expenditures was much higher, at $87.5 \%$ [1]. The World Health Organization (WHO) considers health financing models with high-risk pools, such as health insurance and prepaid schemes, to be a promising means for achieving universal healthcare coverage [2]. The WHO defines risk pooling as "the practice of bringing several risks together for insurance purposes in order to balance the consequences of the realization of each individual risk" [4]. Pooling ensures that the risk related to financing health interventions is borne by all the members of the pool and not by each contributor individually. Risk pooling is required because of the large uncertainty in the magnitude and timing of an individual's health care expenditure needs. It implies 3 redistributive functions: from the rich to the poor, from the healthy to the sick, and from the productive to the unproductive stage of the life cycle [1]. International research shows that the arguments in favor of risk pooling in health care reflect considerations of equity (the transfer of health care resources between individuals) and efficiency (the reduction of individual uncertainty associated with health care needs) $[1,3]$. There are essentially 4 classes of approaches to risk pooling: 1) no risk pool, 2) a unitary risk pool, 3) fragmented risk pools, and 4) in- 
tegrated risk pools, in which fragmented risk pools are compensated for the variations in risk to which they are exposed [4]. To summarize, as risk pooling becomes progressively more integrated, the uncertainty associated with health care expenditures can be reduced. A system of OOP payments exposes individuals to the greatest level of uncertainty, whereas integrated risk pooling seeks to reduce these variations, which are eliminated under a truly unitary system [4].

According WHO's method for the classification of risk-sharing mechanisms in health care financing, WHO's data [3] showed that between 2000-2014 the degree of risk-sharing in low-income countries (mean, 3.22; range, 1.58 to 2.08: of the total 6 points Likert) is low risk-sharing, and in middle-income countries (mean, 1.88; range, 3.04 to 3.48 ) is medium risksharing. This rapid shift in these income countries groups was coincided (2000-2014) with increasing public health expenditure (as a share of total health expenditure) in low-income countries (mean, $41.6 \%$; range, 39.9 to $42.5 \%$ ) and in middleincome countries (mean, $44.6 \%$; range, 45.7 to $50.9 \%$ ), reducing in private health expenditure (as a percentage of total health expenditure) in low-income countries (mean, 58.4; range, 60.1 to $57.5 \%$ ) and middle-income countries (mean, $51.6 \%$; range, 55.2 to $47.7 \%$ ), reducing OOP health expenditure (as a percentage of private expenditure) in low-income countries (mean, $41.6 \%$; range, 45.2 to $37.2 \%$ ), and middle-income countries (mean, $40.9 \%$; range, 45.8 to $36.1 \%$ ). in addition, in time period 2000-2014, external health resources (as a percentage of total expenditure on health) in low-income countries (mean, 27.9\%; range, 16.4 to $33.2 \%$ ), and lower middle-income countries (mean, $0.9 \%$; range, 1.0 to $0.9 \%$ ) had been high increased [3].

Deferent evidence $[1,2,5]$ showed that the combination of different financing mechanisms varies depending on the economic, social, and political conditions in different countries. In the low-income group, the share of the health financing combination is as follows: OOP (67\%), government public resources $(26 \%)$, and private pooling (7\%). With the income level increase in the low income countries and transition to middle income countries, the combination of health financing move towards reducing the share of OOP to $36 \%$ and increasing the share of private pooling up to $10 \%$ and public/governmental resources up to $54 \%$. As income levels in countries rise to a high level, changes in the health financing combination, including a further decline in OOP expenditure up to $14 \%$ and a further increase in private pooling up to $24 \%$ and public/gov- ernmental resources up to $63 \%$ are observed in the high-income countries $[1,2,5]$. In other words, by transferring from the low income group to the middle income group, the share of OOP payment reduce about $31 \%$; whereas, the private pooling and the general government resources increase and $28 \%$, respectively. Moreover, with the transition of countries from the middle income group to high income group, the share of OOP reduce $-22 \%$, and the shares of private pooling and governmental resources increase 13 and $9 \%$, respectively. It should be noted that by transferring countries from the low income group to the high-income group, the percentage changes is as follows: OOP $-54 \%$, private pooling $-17 \%$, and governmental resources $36 \%$ [1].

Furthermore, When health financing systems are aggressive (based on greater risk pooling/sharing, such as public resources and prepayments), the financial risk associated with health care is lower for households, and the pattern of risk-sharing is equitable and efficient. In contrast, regressive health financing systems that rely on direct payments, especially OOP payments, have no risk pooling/sharing and greater financial risks associated with health care for households, making them unequitable and inefficient [1]. Finally, financial health policies including prepayment schemes and the allocation of public resources with higher risk sharing (health-pooled funds), such as universal health coverage among low and middle-income countries, such as Chile, South Korea and Malaysia which have achieved the universal health coverage or are moving rapidly towards it (such as Brazil, Mexico and Thailand), more than $65 \%$ of financing is in the form of pooled financing. The share of health taxes is different in these countries; it is significant in some countries such as Britain, Sweden, Brazil, Malaysia, and Thailand [4]. Therefore, health financing reforms with better risk pooling (such as prepayment schemes, community-based financing, and social health insurance) should be seen as a key strategy for achieving and universal health coverage and reducing the financial barriers to health care in low-income and lower-middle-income countries, such as Africa, south Asia and Middle East regions, resulting in higher utilization but lower OOP expenditures.

\section{ACKNOWLEDGEMENTS}

This study was approved and scientifically supported by the Tarbiat Modarres University (TMU), Tehran, Iran. 


\section{CONFLICT OF INTEREST}

The authors have no conflicts of interest associated with the material presented in this paper.

\section{ORCID}

Ali Ahangar https://orcid.org/0000-0002-7206-0534

Ali Mohammad Ahmadi https://orcid.org/0000-0003-29618202

\section{REFERENCES}

1. Ahangar A, Ahmadi AM, Mozayani AH, Faraji Dizaji S. Transition of health financing models and cost risk sharing in the health sector: a systematic review. Iran Red Crescent Med J 2018 (in press)

2. Preker AS, Carrin G. Health financing for poor people: resource mobilization and risk sharing; 2004 [cited 2018 Jan 9]. Available from: https://openknowledge.worldbank.org/handle /10986/15019.

3. Ahangar A, Ahmadi AM, Mozayani AH, Faraji Dizaji S. The assessment of risk-sharing in the financing of the health sector and health outcomes: a comparative study across WHO's regions [dissertation]. Tehran: Tarbiat Modarres University; 2018.

4. Ahangar A, Ahmadi AM, Mozayani AH, Faraji Dizaji S. Why are risk-pooling and risk-sharing arrangements necessary for financing healthcare and improving health outcomes in low and lower middle- income countries. Health 2018;10(1):122131.

5. Smith PC, Witter SN. Risk pooling in health care financing: the implications for health system performance; 2004 [cited 2018 Jan 9]. Available from: https://openknowledge.worldbank. org/handle/10986/13651. 